\title{
Autocorrelation Kernel Support Vector Machines for Doppler Ultrasound M-Mode Images Denoising
}

\author{
Cristina Soguero-Ruiz ${ }^{1}$, Alicia Guerrero-Curieses ${ }^{1}$, Francisco Javier Palancar ${ }^{1}$, Javier Bermejo ${ }^{2}$, \\ José Carlos Antoranz ${ }^{3}$, José Luis Rojo-Álvarez ${ }^{1}$ \\ ${ }^{1}$ Department of Signal Theory and Communications, Telematics and Computing, \\ Universidad Rey Juan Carlos, Madrid, Spain \\ ${ }^{2}$ Hospital General Universitario Gregorio Marañón de Madrid, Madrid, Spain \\ ${ }^{3}$ Universidad Nacional de Educación a Distancia, Madrid, Spain
}

\begin{abstract}
Doppler ultrasound M-mode images are routinely used in clinical echocardiography, and they have been proposed for non-invasive estimation of the intracardiac pressure gradients in the heart, a process that has been shown to be sensitive to spline interpolation. In this work, we scrutinized the effect of interpolation with a new approach using support vector machines (SVM) for estimation using autocorrelation Mercer kernels in ultrasound images. The SVM algorithm was modified to provide the estimation of a whole image in terms of a reduced set of pixels used as training data set. The autocorrelation of the color Doppler $M$-mode (CDMM) image was estimated with conventional cross-correlation by considering the complete image, and it was used as the Mercer kernel required by SVM. Several subsampling strategies were scrutinized, namely, a heuristic approach, a criterion based on the edges, and a criterion based on the amplitudes. In order to evaluate the proposed methods, we analyzed a previously proposed Doppler image synthetic model, as well as case study with a real image. Results in terms of mean absolute error showed that the minimum error is obtained when information from the edges is considered, yielding 7.37 for single width radial basis function, 2.80 for double with radial basis function, and 0.80 for autocorrelation kernel. The autocorrelation kernel provides with an accurate estimation of the spatiotemporal distribution of flow velocity within the heart using CDMM images. This methodology can be further exploited for enhancing the sensitivity of the pressure gradients estimation to polynomial interpolation and then for improving noninvasive cardiovascular diagnosis.
\end{abstract}

\section{Introduction}

Doppler echocardiography can be considered one of the most useful noninvasive technique to measure blood flow within the heart [1]. Broadly speaking, frequency shift occurs when the ultrasound waves interact with objects in motion, such as red blood cells within blood. This detectable frequency shift is also dependent upon angle of incidence of the ultrasound beams with the blood flow, and it can be used to determine the velocity of the blood flow. Not only the blood velocity, but also intracardiac pressure differences can be obtained noninvasively by using ultrasound images under certain conditions. Historically, Mmode (motion mode) echocardiography, which provides a one-dimensional (depth only) view of the heart, was the first effective modality for the ultrasonic evaluation of the heart. Although newer and more advanced modes of ultrasounds in echocardiography have been developed, and M-mode is a fundamental part of the routine echocardiographic exam and provides an important supplement to the newer echocardiographic modalities.

Previously, the non-invasive estimation of intracardiac pressure gradients has been proposed to be possible from post-processing of Color Doppler M-mode (CDMM) [2]. Whereas this technique has given access to a number of cardiac indices, still its use is mostly based on spline interpolation, which can have a marked smoothing effect on the image preprocessing. In order to estimate the blood velocity from the CDMM images with improved interpolation capabilities, we propose to use a Support Vector Machine (SVM) algorithm with a kernel able to get a better adaptation to this type of images, such as the kernel based on the image autocorrelation function, following the ideas in [3] for interpolating one-dimensional signals. In [4], an SVM algorithm was also used to address this same problem, which was based on a diverse-width Gaussian Mercer kernel, which still can not completely follow the smoothness that characterizes these types of images, as shown in the experiments section.

This paper is organized as follows. Section 2 summarizes the main concepts related to the SVM algorithm with 
autocorrelation kernel, the generated synthetic image and the considered sample selection criteria. Section 3 shows the obtained results when the SVM with autocorrelation kernel is used on synthetic and real CDMM images, and by considering different criteria for sample selection. Conclusions are finally stated in Section 4.

\section{Methods}

SVM with Autocorrelation Kernel. The SVM model for CDMM images estimation [4] uses the following nonlinear regression model. Let $v_{b}(s, t)$ and $\left\{V_{i, j}=\right.$ $\left.v(i \delta s, j \delta t), i=1, \cdots, N_{s}, j=1, \cdots, N_{t}\right\}$ denote the velocity field and the acquired image $\left(N_{s} \times N_{t}\right.$ matrix $)$, respectively. Also, let $[i, j]$ denote the image coordinates of pixel $V_{i, j}$, and let $I$ denote the set of coordinates for all the image pixels. Then, by using some adequate criterion, $I$ can be split into subsets, $I^{\text {train }}$ and $I^{\text {test }}$, to be used for training and testing the model, and hence we have

$$
V_{i, j}=<\boldsymbol{w}, \boldsymbol{\phi}([i, j])>+b+e_{i, j}
$$

with $[i, j] \in I^{\text {train }}$, where $e_{i, j}$ is the model approximation error for the pixel; $\phi([i, j])$ is a nonlinear application of coordinate vector $[i, j]$ to a high-dimensional (say $P$ dimensional) feature space $\mathfrak{F}$; and $b$ is a bias term. A linear regression for the pixel value is given by the dot product of nonlinearly transformed pixel coordinates and $\boldsymbol{w} \in \mathfrak{F}$.

Given this image model, we use the $\varepsilon$-Huber cost proposed in [5], which is a robust cost function that can adapt itself to different kinds of noise. By following the conventional SVM methodology, the previous loss function is regularized with the squared norm of model coefficients and primal problem consists of minimizing

$$
\begin{aligned}
& \frac{1}{2} \sum_{p=1}^{P} w_{p}^{2}+\frac{1}{2 \delta} \sum_{[i, j] \in I_{1}^{\text {train }}}\left(\xi_{i, j}^{2}+\xi_{i, j}^{\star 2}\right)+ \\
& +C \sum_{[i, j] \in I_{2}^{\text {train }}}\left(\xi_{i, j}+\xi_{i, j}^{\star}\right)-\sum_{[i, j] \in I_{2}^{\text {train }}} \frac{\delta C^{2}}{2}
\end{aligned}
$$

with respect to $w^{p},\left\{\xi_{i, j}^{(\star)}\right\}$ (notation for both $\left\{\xi_{i, j}\right\}$ and $\left.\left\{\xi_{i, j}^{\star}\right\}\right)$ and to $b$, and constrained to

$$
\begin{aligned}
V_{i, j}-<\boldsymbol{w}, \phi([i, j])>-b & \leq \varepsilon+\xi_{i, j} \\
-V_{i, j}+<\boldsymbol{w}, \phi([i, j])>+b & \leq \varepsilon+\xi_{i, j}^{\star}
\end{aligned}
$$

and to $\xi_{i, j}, \xi_{i, j}^{\star} \geq 0$, for $[i, j] \in I^{\text {train }}$. In this last equations, $\left\{\xi_{i, j}^{(\star)}\right\}$ are slack variables or losses, and they handle the residuals according to the robust cost function; and $I_{1}^{\text {train }}, I_{2}^{\text {train }}$ are the subsets of pixels for which losses are in the quadratic or in the linear cost zone, respectively.

In brief, by including constraints (3), (4) into (2), the primal-dual functional (or Lagrange functional) is obtained. By making zero the gradient of the Lagrangian with respect to the primal variables [5], and by using the Karush-Khun-Tucker conditions, several manipulations can be done. The correlation matrix of input space pixel pairs can be identified, and denoted as $\boldsymbol{R}([i, j],[k, l]) \equiv<$ $\phi([i, j]), \boldsymbol{\phi}([k, l])>$.

After solving the dual problem in matrix form [5], the velocity for a pixel at $[k, l]$ is given by

$$
\hat{V}_{k, l}=\sum_{[i, j] \in I^{\text {train }}} \beta_{i, j}<\phi([i, j]), \phi([k, l])>+b
$$

with $\beta_{i, j}=\alpha_{i, j}-\alpha_{i, j}^{\star}$, which is a weighted function of the nonlinearly observed times in the feature space. Note that only a reduced subset of the Lagrange multipliers is nonzero, which are called the support vectors, and that the CDMM image estimation is built only with them.

It is known that the calculation of a dot product in $\mathfrak{F}$ can be done with a Mercer kernel. Among the most common Mercer kernels, we find the linear and the Gaussian ones. In [4], the double-width Gaussian kernel was proposed, given by $K_{D}([i, j],[k, l])=\exp \left(\frac{|i-k|^{2}}{-2 \sigma_{s}^{2}}\right) \exp \left(\frac{|j-l|^{2}}{-2 \sigma_{t}^{2}}\right)$, in order to deal with the different scales of the two different dimensions in M-mode, namely, space and time.

Given the smoothness involved in CDMM images, we propose to use a kernel able to adapt to these images, such as the following autocorrelation kernel (adapted from [3]),

$$
K([i, j],[k, l])=\rho_{v}(k-i, l-j)
$$

with

$$
\rho_{v}(m, n)=\sum_{[i, j] \in I^{\text {train }}} V[i, j] \cdot V[i+m, j+n]
$$

and where $m$ and $n$ must be small enough to represent an autocorrelation function with resolution to work with.

The CDMM image model can finally be expressed as

$$
\hat{V}_{k, l}=\sum_{[i, j] \in I^{\text {train }}} \beta_{i, j} K([i, j],[k, l])+b
$$

Cross-validation techniques can be used in order to adjust the free parameters of the SVM cost function $(\varepsilon, \delta, C)$.

Synthetic Image Model. A simple model of diastolic transmitral flow in M-mode color-Doppler was previously created by addition of 3 bivariate Gaussian components, following the methodology in [4], and given by

$$
v_{b}(s, t)=\sum_{i=1}^{3} a_{i} \exp \left\{-\frac{1}{2}\left[s_{i}, t_{i}\right] \Sigma_{i}^{-1}\left[s_{i}, t_{i}\right]^{T}\right\}
$$

where $\left[s_{i}, t_{i}\right]$ denotes a bidimensional row vector, $\Sigma_{i}$ is the covariance matrix of each component. As shown in Fig. 1. two components account for early LV filling (E-wave, $i=1,2$ ), and a lower amplitude component emulates late 

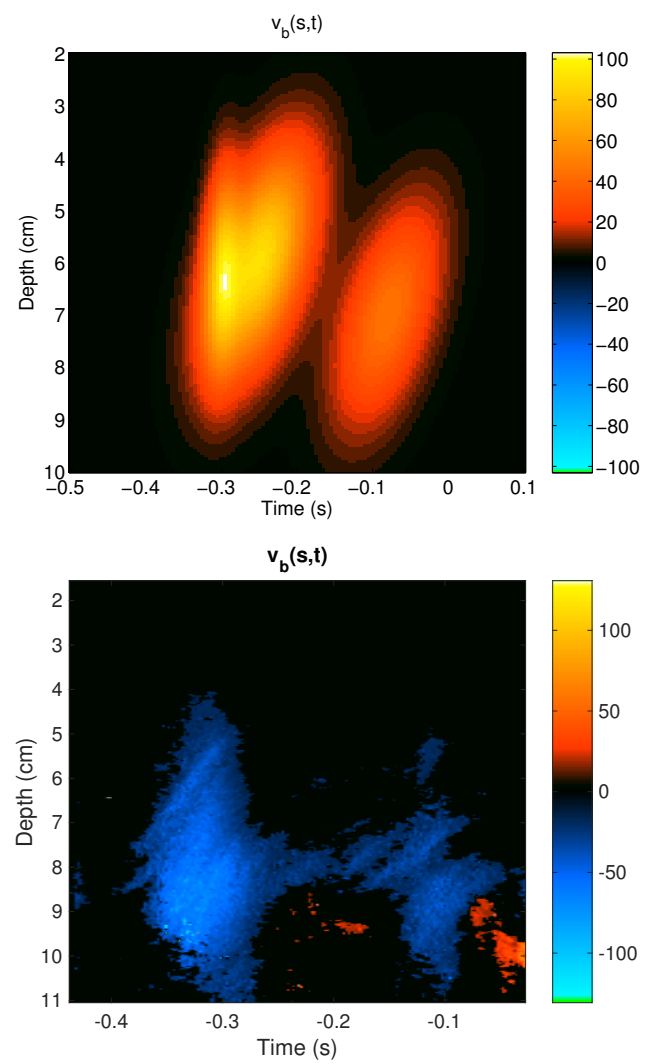

Figure 1. Synthetic (up) and real (down) CDMM image.

filling (A-wave, $i=3$ ). Time 0 was defined at the QRS onset

Sample Selection Criteria. The training and test sets, used by the SVM machine to rebuild the total CDMM images, have been scrutinized according to different criteria of sample selection, as described next:

1. Random: The training and test samples are selected from the image randomly.

2. Edge Based: By using a 2-D edge filter (Sobel filter [6]), only some of those samples from image regions with sharp amplitude changes are considered to build the machine. A threshold can be considered to obtain a training set with a large enough number of samples. Finally, the training and test sets are generated.

3. Amplitude Based: The image pixels are ranked in order of its amplitude from greatest to least. Then, by using a Gaussian function, larger weights are given to those samples (pixels) with larger amplitudes. Finally, the training and test sets are generated.

\section{Experiments and Results}

In this section, results with the Doppler image synthetic model and with a real image are presented. For the synthetic image, different approaches to SVM algorithm with
Table 1. Synthetic image. MAE in CDMMI approximation considering different kernels.

\begin{tabular}{c|c|c|c}
\hline \# tr. pixels & RBF & D-RBF & Corr \\
\hline \hline $\mathbf{2 5 0}$ & 7.93 & 1.52 & 1.23 \\
\hline $\mathbf{1 0 0 0}$ & 7.01 & 0.75 & 0.72 \\
\hline
\end{tabular}

Table 2. Synthetic image. MAE in CDMMI approximation considering different sample selection criteria.

\begin{tabular}{c|c|c|c}
\hline \# tr. pixels & Random & Edges & Amplitude \\
\hline \hline $\mathbf{1 0 0}$ & 1.86 & 1.87 & 4.15 \\
\hline $\mathbf{2 5 0}$ & 1.23 & 1.13 & 3.09 \\
\hline $\mathbf{5 0 0}$ & 0.95 & 0.90 & 1.95 \\
\hline $\mathbf{1 0 0 0}$ & 0.82 & 0.74 & 1.76 \\
\hline
\end{tabular}

three different kernels were used for denoising. For both images, the training subset (random image subsampling) were selected following different sample selection criteria and considering different number of training samples. Mean absolute error (MAE) of CDMM image approximations was calculated in the test pixels.

\subsection{Results on Synthetic Data}

Kernel Evaluation. For a fair comparison, a SVM algorithm was used with RBF, D-RBF and autocorrelation kernels, considering the same number of training samples (250 and 1000) and a random sample selection criteria. Results in terms of MAE (see Table 1) show that better approximations are obtained when an autocorrelation kernel is used and when the number of training sample increases.

Sample Selection Criteria Evaluation. From Table 1 we concluded that using an autocorrelation kernel provides better CDMM image approximation. Then, we evaluated the impact of using different sample selection criteria and different number of pixels in the training subset. Results in terms of MAE are shown in Table 2. For all criteria, lower MAE values were obtained as the number of training samples was higher. Regarding the selection criteria, the approach based on edges provides the best CDMM image approximation independently of the number of samples considered.

\subsection{Results in a Real Image}

A CDMM image from a healthy volunteer is also analyzed in this work. The $126 \times 171$ image was subsampled by 2 . Table 3 presents the MAE obtained when considering different sample selection criteria and different number of pixels with the autocorrelation kernel, noting that we increased here the number of training points. We can obtain similar conclusions as with the synthetic image. As expected, the higher the number of training samples, the 
Table 3. Real image. MAE in CDMMI approximation considering different sample selection criteria.

\begin{tabular}{c|c|c|c}
\hline \# tr. pixels & Random & Edges & Amplitude \\
\hline \hline $\mathbf{1 0 0}$ & 4.28 & 4.22 & 16.29 \\
\hline $\mathbf{1 0 0 0}$ & 2.25 & 2.53 & 6.81 \\
\hline $\mathbf{2 0 0 0}$ & 2.22 & 2.19 & 5.85 \\
\hline $\mathbf{3 0 0 0}$ & 2.02 & 2.00 & 4.94 \\
\hline
\end{tabular}

better approximations. Figure 2 shows the spatial distribution of the CDMMI residuals when using 3000 training points and edges vs. amplitudes sample selection criteria.

\section{Conclusions}

Different approaches for CDMM image denoising have been proposed and scrutinized. The methods used in this work are based on non-linear SVM with single and double width radial basis functions, and autocorrelation kernel, considering different selection criteria and different number of samples. Comparisons among them show that the performance of SVM using an autocorrelation kernel with a selection criteria based on amplitudes provides with the best results. Moreover, we can conclude that the higher the number of training samples, the better the SVM performance, though a limited set yields a good enough quality. Oncoming work is devoted to extend this work to intracardiac pressure gradients which are usually estimated by post-processing of flow CDMM images.

\section{Acknowledgements}

This work has been partly supported by Spanish Projects TEC2016-75161-C2-1-4 and TEC2013-48439C4-1-R. CSR is supported by FPU grant AP2012-4225. Thanks to Noelia de Pablo for his preliminary work on experiments with autocorrelation kernel.

\section{References}

[1] Weyman AE. Principles and practice of echocardiography. Lea Febiger 2nd edition 1994;.

[2] Yotti R, Bermejo J, Antoranz JC, Desco MM, Cortina C, Rojo-Álvarez JL, Allué C, Martín L, Moreno M, Serrano JA, et al. A noninvasive method for assessing impaired diastolic suction in patients with dilated cardiomyopathy. Circulation 2005;112(19):2921-2929.

[3] Figueras C, Barquero-Pérez O, Rojo-Álvarez JL, MartínezRamón M, Guerrero-Curieses A, Caamaño A. Spectrally adapted Mercer kernels for support vector nonuniform interpolation. Signal Processing 2014;94:421-433.

[4] Conde-Pardo P, Guerrero-Curieses A, Rojo-Álvarez J, Yotti R, Requena J, Antoranz J, Bermejo J. A new method for single-step robust post-processing of flow color doppler M-
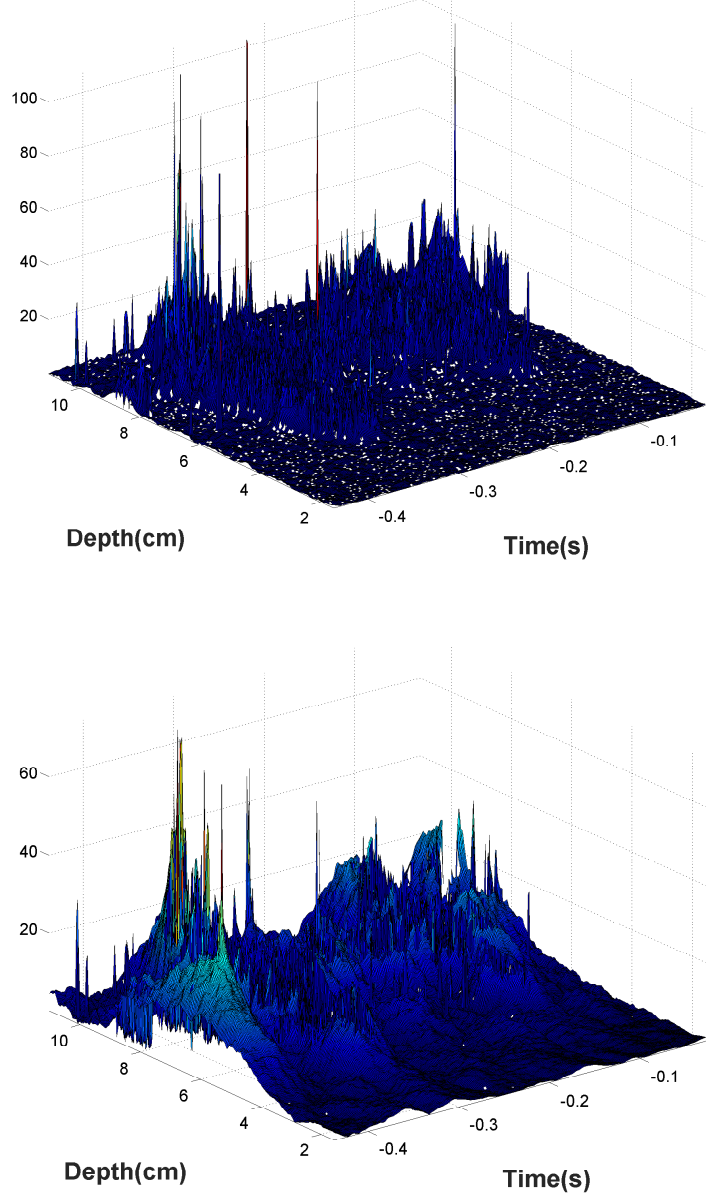

Figure 2. Color Doppler M-mode trasmitral flow velocity recording for a healthy volunteer: residuals when using sample selection criteria based on edges (up) and amplitude (down).

Mode images using support vector machines. In Computing in Cardiology. Valencia, Spain, Sep 2006; 525-528.

[5] Rojo-Álvarez JL, Martínez-Ramón M, Figueiras-Vidal A, dePrado Cumplido M, Artés-Rodríguez A. Support vector method for ARMA system identification. IEEE Trans on Signal Processing 2004;52(1):155-164.

[6] Farid H, Simoncelli E. Differentiation of discrete multidimensional signals. IEEE Trans on Image Processing 2004; 13(4):496-508.

Address for correspondence:

C Soguero-Ruiz

University Rey Juan Carlos. D201, Camino del Molino s/n

28943 - Fuenlabrada (Madrid), Spain

Phone: +3491488 8462

cristina.soguero@urjc.es 